\title{
INCLUSIONES PIROCLÁSTICAS EN PASTAS CERÁMICAS DEL VALLE DE TAFÍ: IMPLICANCIAS PARA LAS PRÁCTICAS DE PRODUCCIÓN
}

\author{
María Cecilia Páez y Marcelo Arnosio ${ }^{2}$
}

\section{$*$ Introducción}

Resumen

El uso de inclusiones pumíceas como antiplástico es una práctica alfarera asociada a los momentos tardíos e incas del Noroeste Argentino. El estudio de su naturaleza como del proceso tecnológico por el cual se incorporan a las pastas puede conducir a los significados sociales y políticos de dicha práctica. Análisis de pasta submacroscópicos y microscópicos aplicados en fragmentos de dos sitios del valle de Tafí determinaron que la fracción clástica más fina de las pastas corresponde a material piroclástico de depósitos de caída, los que se encuentran en distintos espacios de la región. Los alfareros prehispánicos identificaron, seleccionaron y transportaron estos materiales para preparar las pastas, aparentemente sin previo tratamiento de molienda o selección

de sus componentes. La similitud de la alfarería con este tipo de inclusiones en todo el Noroeste Argentino sugiere ciertas normas para manufacturar las piezas, las que se vincularían con el dominio del Inka.

Palabras claves: alfarería - antiplástico - período Inca - Tafí - Noroeste

Argentino.

Abstract

The use of pumiceous inclusions as coarse temper is a practice recorded in pottery associated with Late and Inca periods in Northwest

Argentina. The nature of these inclusions and the technological

process that incorporates them into pottery provide clues on the social and political significance of this practice. Sub-macroscopic and microscopic paste analysis carried out on fragments obtained from two Tafí valley sites, lead to conclude that the finer clastic fractions composing the pastes come from pyroclastic-fall deposits available in different areas of Northwest Argentina. Pre-Columbian potters identified, selected and transported these materials in order to prepare ceramic pastes, without need to grind or select them. Similar pottery inclusions found throughout the region suggest a shared manufactur-

ing pattern related to Inka rule.

Key words: pottery - temper - Inca period - Tafí - Northwest Argentine.

Recibido: septiembre 2008. Aceptado: mayo 2009.

1 CONICET. Paseo del Bosque s/nº. C.P. 1900, La Plata.ARGENTINA.Email: ceciliapaez@gmail.com

2 Instituto GEONORTE, Universidad Nacional de Salta. Av. Bolivia 5151. C.P. 440o, Salta, ARGENTINA. Email: marnosio@unsa.edu.ar
Las pastas cerámicas con inclusiones blancas se vinculan a la tecnología de producción desarrollada durante la expansión incaica en el Noroeste Argentino. Requirió un especial cuidado, tanto en la selección de las materias primas como en la preparación de las pastas, otorgando al objeto terminado una característica distintiva asocia$\mathrm{da}$ a ciertos estilos decorativos y excluyente de otros. Sin embargo, más allá de su importancia como técnica, esta manufactura cerámica contribuyó a producir significados al formar parte activa de los procesos sociopolíticos regionales relacionados al Estado inca en espacios alejados de su centro político en Cusco.

Inicialmente fue identificada en el oeste catamarqueño (Cremonte 1991), aunque a partir de entonces se le reconoce una amplia dispersión en sitios incas del Noroeste Argentino. Esta dispersión, sumada a la similitud en la naturaleza y características de la materia prima de las inclusiones, indica una práctica de manufactura extendida que permite caracterizar un momento del desarrollo sociocultural prehispánico. En este sentido, es importante determinar la forma en que efectivamente las inclusiones se integraron a las pastas, pudiendo ser parte de las arcillas utilizadas en la confección de las piezas, o mediando algún proceso de extracción, selección y/o procesamiento de las inclusiones previo a su incorporación en la cerámica.

En este trabajo exploramos tales aspectos a partir de fragmentos obtenidos de excavaciones sistemáticas realizadas en sitios tardíos e incas en el valle de Tafí (Manasse 
1995-96), para resolver dos aspectos fundamentales: la naturaleza y características de estas inclusiones para el valle, y las particularidades del proceso tecnológico que permitió su incorporación en las pastas. Los resultados constituirán un aporte al conocimiento de la dinámica social local y regional, y deberán profundizarse y discutirse en el marco de las interpretaciones de la política estatal incaica en el Noroeste Argentino; y, aunque no esperamos que nuestras interpretaciones se extiendan al resto de la alfarería con inclusiones blancas, creemos que es importante poner en discusión una técnica inédita de preparación de las pastas.

\section{* Contextualización Regional de la problemática}

El análisis de un conjunto cerámico procedente de los sitios incaicos Potrero Chaquiago e Ingenio del Arenal Médanos, ambos en Catamarca, puso en evidencia la presencia de inclusiones blancas en las pastas de fragmentos Rojo sobre Blanco, Negro sobre Rojo, Cusco Policromo y uno "posiblemente altiplánico o incaico" (Cremonte 1991). Estas inclusiones se encontraban en densidades importantes (18 a 30\%), caracterizadas por su forma redondeada. Los análisis determinaron que correspondían a gránulos de vidrio volcánico, incorporados a las pastas a partir del molido de rocas volcánicas blandas de tipo pumíceas (Cremonte 1994).

La similitud visual -aunque no petrográfica- con las pastas altiplánicas o puneñas de la región Chicha -que en este caso incluían lutitas (Krapovickas 1975; Cremonte et al. 2007) - llevó a pensar en la presencia de mitmaqkuna altiplánicos en la subárea valliserrana. Estos grupos, que habrían fabricado cerámica con antiplástico de lutitas para el Estado inca en el norte puneño, habrían continuado sus propias tradiciones de manufactura alfarera, aunque con una materia prima diferente, no ya lutitas sino material piroclástico (Cremonte 1994).

Posteriores investigaciones en el Noroeste Argentino encontraron esta característica tecnológica en la cerámica de diversos sitios, tanto de la subárea valliserrana como de la Puna (Figura 1). Si bien los sitios en los que se ha identificado hasta el momento se encuentran en el actual territorio argentino, es probable que pueda hallarse fue-

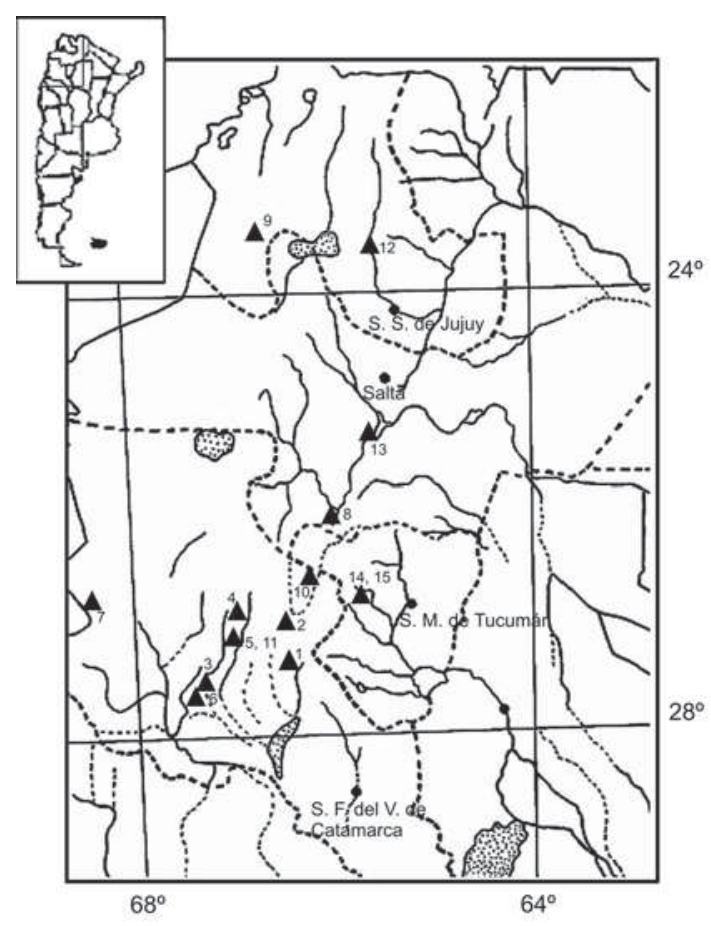

Figura 1. Distribución de sitios arqueológicos con alfarería con inclusiones pumíceas en el Noroeste Argeentino. 1) Potrero Chaquiago (Catamarca); 2) Ingenio del Arenal Médanos (Catamarca); 3) El Shincal (Catamarca);4) La Angostura (Catamarca); 5) Hualfín Inca (Catamarca); 6) Tambillo (Catamarca); 7) Tambo San Francisco (Catamarca); 8) Valle Calchaquí (Salta); 9) Susques (Jujuy); 10) Fuerte Quemado (Catamarca); 11) San Fernando del valle de Hualfín (Catamarca); 12) Quebrada de Humahuaca (Jujuy); 13) Cabra Corral (Salta); 14) LCZVIIISı (Tafí del Valle, Tucumán); 15) LC(1) (Tafí del Valle, Tucumán).

ra de sus límites, si se considera la distribución del arco volcánico y las fronteras prehispánicas.

El sitio El Shincal, en la localidad de Londres (depto. Belén, Catamarca), con su arquitectura manifiestamente incaica, con varias kallanka, un ushnu y una gran plaza de $175 \mathrm{~m}$ de largo, son el centro de un asentamiento que tuvo funciones estatales de carácter público y que se considera uno de los más destacados del Noroeste Argentino (Raffino 2004). Análisis de pasta realizados en fragmentos recuperados de este sitio determinaron la presencia de trozos pumíceos en densidades altas en piezas estatales y otras asociadas al Tardío local. ${ }^{3}$ Esta

3 También denominado Agroalfarero Tardío en el Noroeste Argentino (A. R. González 1963) e Intermedio Tardío en el norte de Chile (Schiappacasse et al. 1989). 
cerámica representa un porcentaje importante de la alfarería del sitio (Giovannetti com. pers. 2008). En una región relativamente próxima, el bolsón de Belén, estas mismas inclusiones se identificaron en fragmentos de estilo Belén que pudieron correlacionarse con recursos disponibles localmente (Puente $2008 \mathrm{Ms}$ ). Otros sitios próximos son Tambillos, a $16 \mathrm{~km}$ de El Shincal y Hualfín Inca, ambos asociados a la presencia estatal (Lynch com. pers. 2009; Moralejo com. pers. 2009). También se identificaron pretrográficamente gránulos de vidrio volcánico en densidades importantes en fragmentos incaicos del Tambo San Francisco, un sitio inca localizado en las proximidades de la frontera con Chile, en el oeste catamarqueño, y en sitios del valle Calchaquí, en Salta (Williams 2003 Ms; De la Fuente 2007.

Uno de los sitios más septentrionales con alfarería que presenta estas pastas se encuentra en las proximidades de Susques, en la Puna jujeña, de donde procede un cuello de aríbalo Inca cusqueño. Los análisis petrográficos realizados sobre este fragmento indicaron una composición pumícea significativa, obtenida a partir del molido intencional de material vítreo volcánico del tipo de las ignimbritas o tobas soldadas. El contenido de diatomeas indican un origen alóctono para el fragmento, posiblemente relacionado con el lago Titicaca, algunos lagos del centro de Perú, y el lago Chungara en el norte de Chile (Solá y Morales 2007).

Además de estos materiales que hemos conocido por referencias bibliográficas o de comunicaciones personales, hemos registrado piezas con estas inclusiones en distintos museos de Argentina. ${ }^{4}$ Si bien no fue posible realizar análisis específicos para obtener una mayor confiabilidad en nuestras observaciones, justamente por tratarse de colecciones museísticas, sí pudimos identificar inclusiones blancas en platos incaicos del Noroeste Argentino. En el Museo de Ciencias Naturales de la Universidad Nacional de La Plata identificamos ejemplares procedentes de Fuerte Quemado (depto. Santa María, Catamarca), San Fernando del valle de Hualfín (depto. Belén, Catamarca) y Quebrada de Humahuaca (Jujuy). En el Museo de Sitio El Shincal (depto. Belén,

\footnotetext{
4 Este relevamiento se hizo con objetivos específicos, por lo que no tuvimos en cuenta todas las formas incaicas, sino solamente la forma de plato (Páez y Giovannetti 2008).
}

Catamarca) -sitio para el cual ya teníamos información sobre una significativa presencia de estas inclusiones-, también registramos piezas completas que lo comprobaron, al igual que en el Museo Antropológico de Salta, donde estaba depositado un apéndice zoomorfo de plato proveniente de Cabra Corral (valle de Lerma, Salta).

Estos datos amplían el panorama distribucional de este atributo tecnológico a las actuales provincias de Jujuy, Salta, Catamarca y Tucumán. En esta última, particularmente, se ha detectado en el valle de Tafí, en cuyos sitios nos centraremos a continuación.

\section{* El valle de tafí}

Este valle constituyó un espacio significativo para el asentamiento humano prehispánico debido a que articula dos espacios geográficamente diferentes: los valles del este, áridos, de clima cálido-templado, y la llanura oriental, cálida y húmeda, con ambiente de bosques y selvas. Se localiza en el borde oriental de la subárea valliserrana, al oeste de la provincia de Tucumán, entre $26^{\circ} 45^{\prime}$ y $26^{\circ} 58^{\prime}$ Lat. S y $65^{\circ} 37^{\prime}$ y $65^{\circ} 52^{\prime}$ Long. O. Se trata de una cuenca intermontana a 2000 m.snm, que separa dos sistemas montañosos importantes: las cumbres calchaquíes, al norte, y las sierras de Aconquija, al sur; y que además, conecta los valles calchaquíes, al oeste, con la llanura chacopampeana, al este (Figura 2).

Las evidencias arqueológicas señalan una ocupación importante tanto en términos espaciales como cronológicos (Manasse 2001). Si bien el referente cultural local han sido las sociedades formativas que dejaron notables vestigios de arquitectura en piedra, son cada vez más frecuentes las depresiones cuadrangulares y la alfarería tardía y del período de expansión incaica. Abundan las clásicas "urnas santamarianas", ampliamente referidas en la literatura arqueológica del Noroeste Argentino (Ambrosetti 1908; Bregante 1926; Perrota y Podestá 1978; Weber 1978, entre otros), caracterizando el desarrollo estilístico del valle desde 9oo DC hasta la llegada de los incas, e incluso, las últimas manifestaciones del estilo serían contemporáneas al Estado. Esta alfarería se caracteriza por el uso de dos o tres colores (negro sobre blanco; negro y rojo sobre blanco); la trí- 


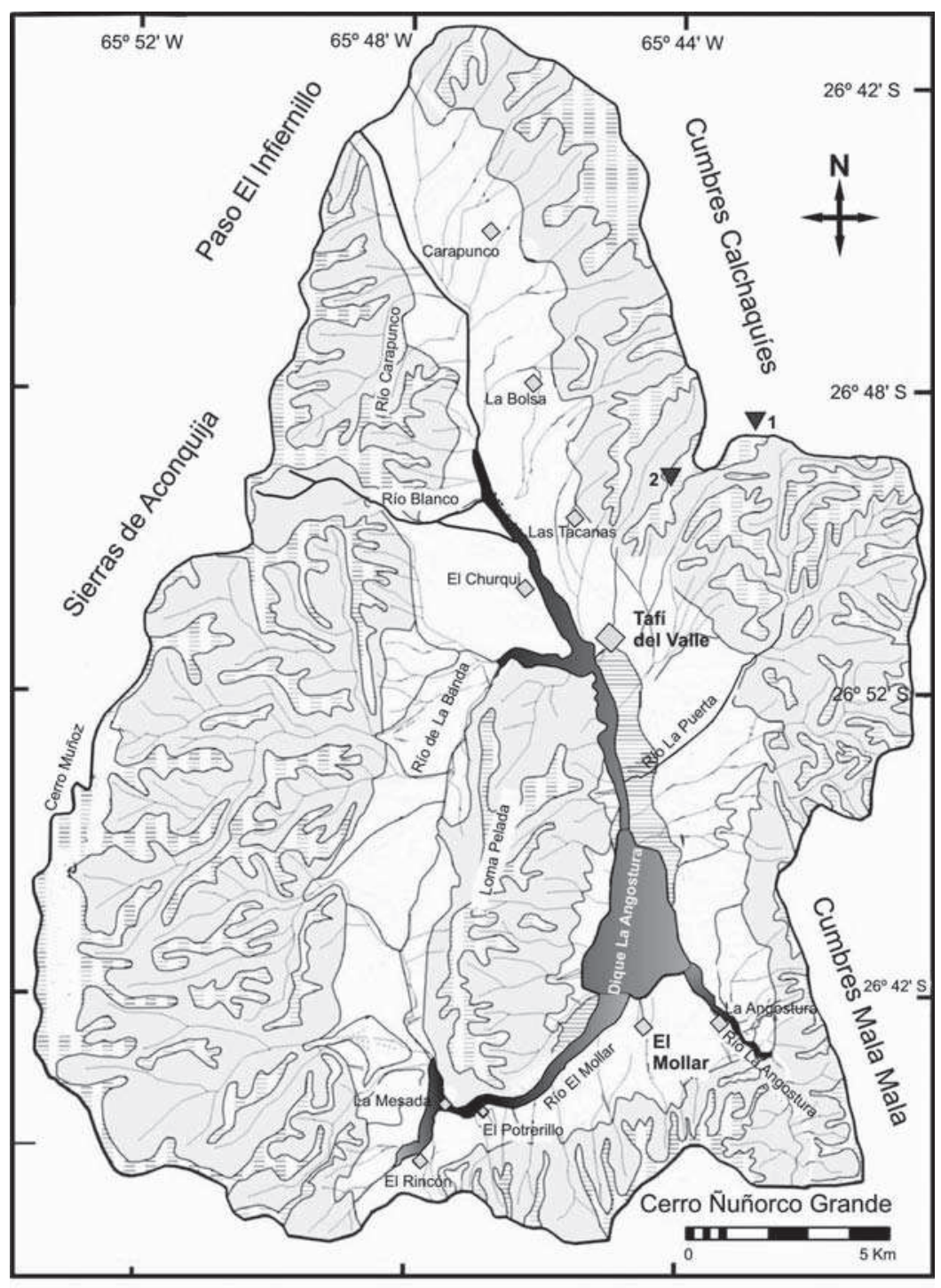

ק1. Sitio LCZVIIIS1

2. SitioLC (1)

Figura 2. El valle de Tafí.

croma se asocia a momentos más tempranos del desarrollo de dicha entidad cultural. Los motivos son tanto geométricos como figurativos, destacando la representación antropomorfa del rostro que adquiere distintas características. De acuerdo al análisis de los rasgos morfológicos y decorativos de ejemplares completos de museos, y a las modificaciones de estos rasgos, se llegó a definir una serie de fases que abarcan desde el surgimiento hasta la disolución de la sociedad santamariana (Perrota y Podestá 1973 Ms, 1978; Weber 1978). Esta alfarería se asocia tradicionalmente al entierro de niños, aunque no de forma exclusiva, estando en muchos contextos acompañada de pucos decorados. En momentos tardíos del desarrollo prehispánico del valle, se vincula también al estilo Famabalasto Negro Grabado, originalmente definido en el sitio homónimo, al igual que el estilo santamariano, en el valle de Santa María.

En el valle también se encuentra alfarería conocida estilísticamente como Belén, definida inicialmente para

\footnotetext{
No38/2009

Estudios ATACAMEÑos

Arqueología y Antropología Surandinas
} 
el centro y oeste de la provincia de Catamarca (Bregante 1926; Bennett et al. 1948; González 1955). En urnas y pucos destaca la decoración en negro sobre rojo, con diseños geométricos y figurativos, a veces recurriendo también a las excisiones. Hasta el momento no es del todo claro el momento en que las sociedades portadoras de esta alfarería llegan al valle, aunque algunos autores han planteado que ocurrió junto con la reorganización poblacional llevada a cabo por los incas en este territorio (Sempé 1999, 2006).

El uso de dos colores en el estilo santamariano también incluye el negro sobre rojo, una modalidad alfarera que combina características morfológicas e iconográficas del estilo santamariano clásico y aquellas del estilo Belén. Estas piezas se asociarían al momento de la expansión inca perdurando probablemente hasta tiempos posteriores, tal como se desprende de los análisis contextuales (Tarragó 2000; Marchegiani et al. 2007).

La presencia incaica en el valle también está representada por abundante platos, aribaloides y ollas con pedestal que alcanzan una importante representación, así como aquellos estilos contemporáneos, entre los que se cuentan el Famabalasto Negro sobre Rojo y Yocavil (Páez y Patané 2007; Manasse y Páez 2008).

Todas las evidencias anteriores junto a la arquitectura, uso del espacio, metalurgia y funebria, entre otras, indican la importancia de los procesos que contribuyeron a configurar la historia social del valle y a través de los cuales se habrían reproducido los significados al interior de la sociedad. Esto en referencia no solo al valor de la cerámica en tanto reflejo de una estructura sociopolítica e ideológica, sino también en su protagonismo en la performance de la estructura y funcionamiento de la vida social (Manasse et al. 2006; Manasse y Páez 2008; Páez 2008 Ms).

\section{* Metodología de anÁlisis}

La muestra analizada está formada por 20 fragmentos procedentes de los sitios LCZVIIIS I (Pucara de las Lomas Verdes), y LC(1) o Chasco Álvarez, ambos ubicados en la localidad de Los Cuartos en Tafí del Valle. El primero se emplaza en las laderas meridionales de las cumbres calchaquíes, a $2410 \mathrm{~m} . s n m$ (Santillán de Andrés 1951; Manasse 2003); el segundo se ubica en el sector bajo del valle, y ambos se asocian al momento de expansión incaica (Manasse 2003; Patané 2008).

Los fragmentos se seleccionaron de una muestra más amplia que incluía fragmentos Santa María (Negro sobre Blanco y Negro sobre Rojo), Belén, Famabalasto (Negro Grabado y Negro sobre Rojo), Inca y una importante proporción de materiales toscos que, a partir de las características morfológicas y de pasta, pudieron en gran parte asociarse a los momentos tardíos e inca. Del sitio LCZVIIISi se analizaron 11 fragmentos procedentes de excavaciones sistemáticas de las estructuras 18 y 27 (Patané 2008), en tanto el resto provenía de un rescate arqueológico en el sitio LC(1) (Manasse 2003). Todos ellos presentan pastas con inclusiones blancas (Tabla 1).

Las inclusiones analizadas son visibles macroscópicamente en las fracturas de los fragmentos, y cuando los engobes y/o pinturas están desgastados, también en las superficies (Figura 3). Por eso realizamos una primera caracterización general del conjunto considerando los aspectos tecnológicos y morfoestilísticos. A partir de esto, utilizamos metodologías específicas para el análisis de las pastas, realizando observaciones con lupa binocular de bajos aumentos (20X-40X) para una caracterización general del fragmento en cuanto a las tres categorías componentes de la pasta (matriz arcillosa, inclusiones no plásticas y espacios vacíos o cavidades) y sus relaciones.

La información que se obtuvo utilizando este grado de detalle debió complementarse con la que proporciona la microscopía tanto petrográfica como estereoscópica. Con estas observaciones se pudieron identificar las inclusiones y realizar algunas aproximaciones a su génesis. Complementariamente, esto último debió abordarse con información geológica específica. En este sentido, nos basamos en la petrografía de depósitos piroclásticos seleccionados, localizados relativamente cerca de nuestra área de estudio, en el Noroeste Argentino. En conjunto, todas estas técnicas proporcionaron un cuerpo de datos significativo a partir del cual fue posible realizar inferencias sobre el proceso tecnológico de manufactura de las piezas (ver Figura 3).

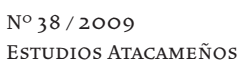




\begin{tabular}{|c|c|c|c|}
\hline Identificación & Sitio & Asociación estilística & Identificación morfológica \\
\hline $\mathrm{LC} 5 \mathrm{O}-\mathrm{P}-15$ & LCZVIIIS $_{1}$ & Santa María N/Bl & Puco \\
\hline $\mathrm{LC}_{54 \mathrm{a} 21}$ & LCZVIIIS $_{1}$ & Santa María N/R & Urna \\
\hline $\mathrm{LC}_{54} \mathrm{ar}_{62}$ & LCZVIIIS $_{1}$ & Santa María N/Bl & Puco \\
\hline $\mathrm{LC}_{54} \mathrm{a}_{3} 8$ & LCZVIIIS $_{1}$ & Belén o Inca & Forma cerrada* \\
\hline $\mathrm{LC}_{54} \mathrm{a}_{37}$ & LCZVIIIS $_{1}$ & Belén o Inca & Indeterminada \\
\hline LC61b29 & LCZVIIIS $_{1}$ & Santa María N/R & Urna \\
\hline LC61b8 & LCZVIIISI $_{1}$ & Santa María N/R & Urna \\
\hline LC61b55 & LCZVIIIS $_{1}$ & Santa María N/R & Urna \\
\hline LC61b59 & LCZVIIIS $_{1}$ & Santa María N/R & Urna \\
\hline LC61b61 & LCZVIIIS $_{1}$ & Santa María N/R & Urna \\
\hline LC61b24 & LCZVIIIS $_{1}$ & Santa María N/R & Urna \\
\hline $\mathrm{LC}(1) 26$ & $\mathrm{LC}(\mathrm{l})$ & Inca & Plato \\
\hline LC(1) 117 & $\mathrm{LC}(1)$ & Santa María N/Bl & Urna \\
\hline $\mathrm{LC}(1)_{94}$ & $\mathrm{LC}(1)$ & Inca & Aríbalo \\
\hline $\mathrm{LC}(1)_{705}$ & $\mathrm{LC}(1)$ & Inca & Plato \\
\hline $\mathrm{LC}(\mathrm{I}) 250$ & $\mathrm{LC}(\mathrm{l})$ & Inca & Plato \\
\hline $\mathrm{LC}(1)_{23}$ & $\mathrm{LC}(\mathrm{l})$ & Inca & Plato \\
\hline $\mathrm{LC}(1)_{19}$ & $\mathrm{LC}(1)$ & Santa María N/R & Urna \\
\hline $\mathrm{LC}(1) 8$ & $\mathrm{LC}(\mathrm{I})$ & Santa María N/R & Urna \\
\hline $\mathrm{LC}(1)_{740}$ & $\mathrm{LC}(\mathrm{l})$ & Santa María N/R & Urna \\
\hline
\end{tabular}

Tabla 1. Fragmentos analizados submacroscópica y microscópicamente. N/Bl: Negro sobre Blanco; N/R: Negro sobre Rojo. *Se utilizó la categoría "forma cerrada" para este fragmento, ya que solo pudo asignarse a esta clasificación más abarcativa (abierta/cerrada), siendo imposible lograr identificaciones más precisas dentro de estos dos grupos. Se utilizó la categoría "indeterminado" para los casos en que no fue posible realizar ninguna aproximación morfológica.

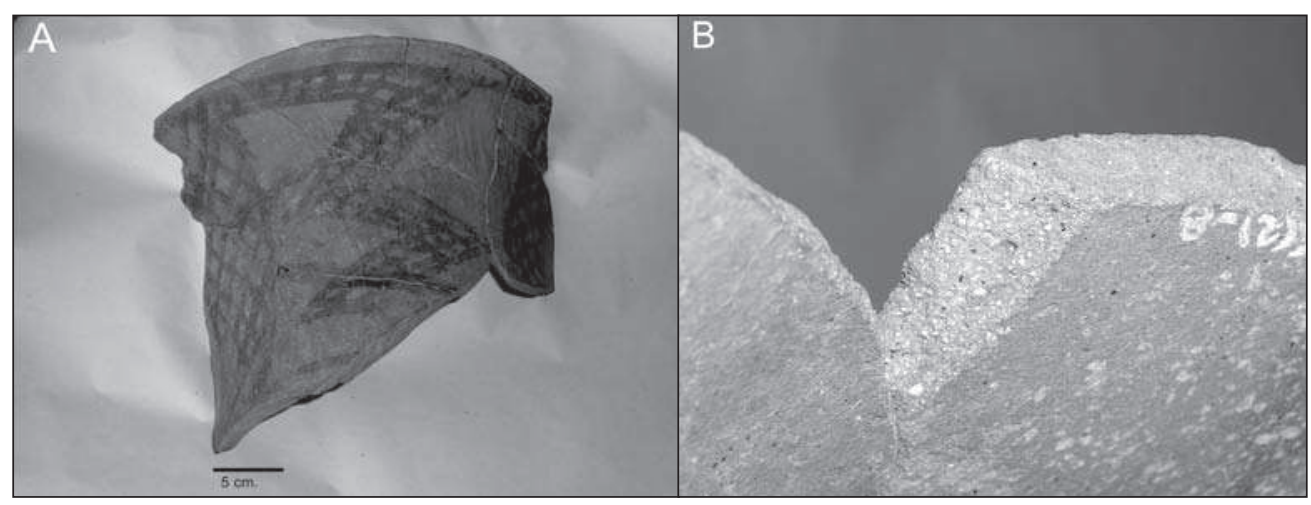

Figura 3. a) Cuello de urna Santa María Negro sobre Rojo del sitio LC(1); b) Detalle del corte fresco del fragmento donde pueden observarse las inclusiones pumíceas, como puntos blancos en la pasta. 


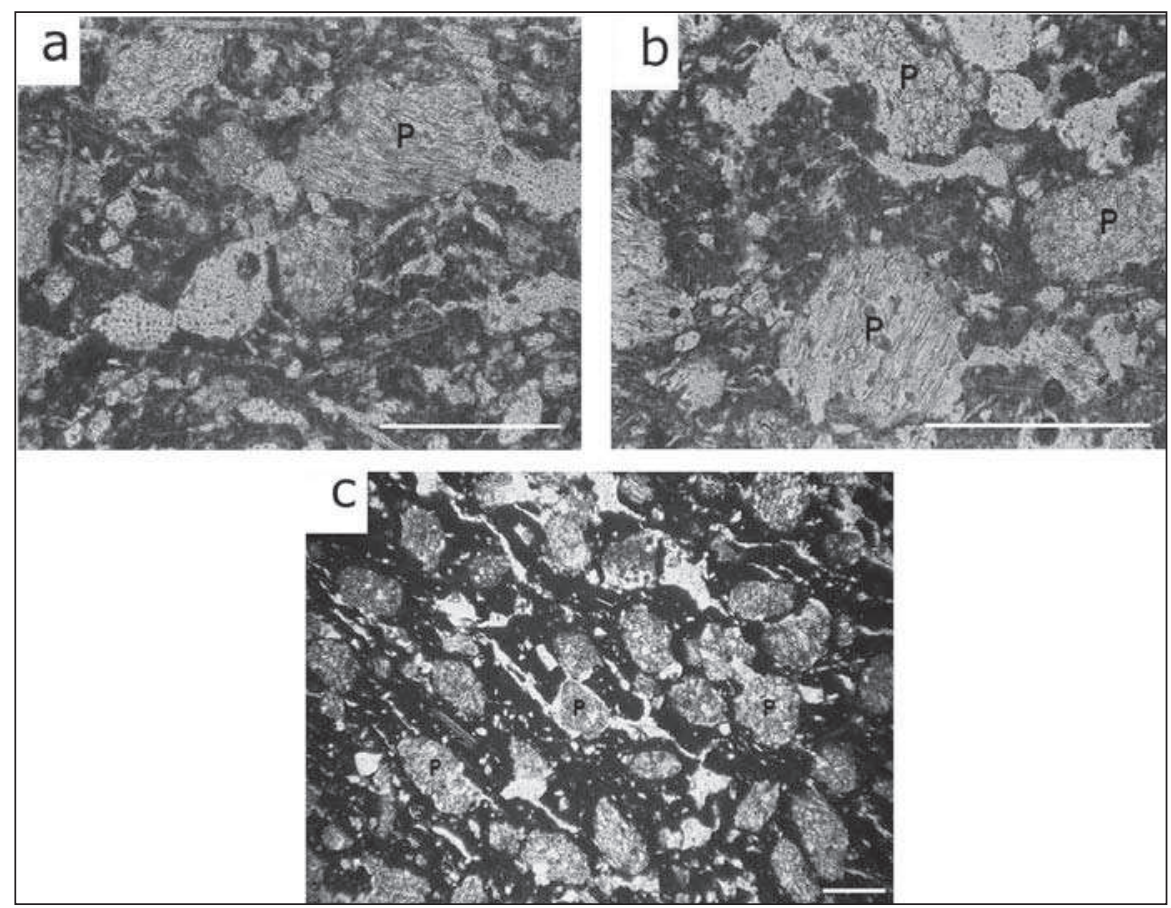

Figura 4. Inclusiones pumíceas observadas con microscopio petrográfico. Formas de las vesículas:

a) Tubulares (fragmento LC54a37); b) Tubulares y esféricas (fragmento LC(1)250);

c) Detalle de la pasta del fragmento LC54a21, donde se observa la densidad y características de forma y tamaño de las pómez (P). Escala $1 \mathrm{~mm}$.

\section{* Fragmentos pumíceos}

El análisis de los fragmentos realizado con distintas metodologías permitió asociar las inclusiones a componentes piroclásticos, específicamente pumíceos o vidrio volcánico vesiculado, presentes en las pastas en porcentajes muy superiores al del resto de los componentes no plásticos.

La altavesicularidad notoria a través delos análisis microscópicos y de las observaciones en lupa binocular (40X) es una característica compartida por las inclusiones de las 20 muestras analizadas. Identificamos formas de vesículas tubulares y esféricas en fragmentos separados, o a escasa distancia en una misma inclusión (Figuras 4a y 4b). En todos los casos el tamaño de las vesículas es variable. También se observaron al interior de los fragmentos pumíceos minerales de distinto tamaño, aunque en densidades bajas.
Estas inclusiones no presentan en ningún caso angulosidad, siendo por el contrario, redondeadas o subredondeadas. ${ }^{5}$ Los tamaños varían entre $0.25 \mathrm{~mm}$ y $0.50 \mathrm{~mm}$ (tamaño medio) y $0.50 \mathrm{~mm}$ a $1 \mathrm{~mm}$ (tamaño grueso), con marcada homogeneidad en sus proporciones en todos los casos analizados, no registrando en ninguno tamaños inferiores o superiores a los mencionados. Así, se obtuvo una selección relativamente buena para este tipo de componentes (Figura 4c).

Como ya dijimos, la densidad es uno de los rasgos que más llama la atención, superando cualquiera de los otros componentes no plásticos. La cuantificación realizada con las observaciones en lupa binocular sugiere un amplio predominio de las pómez por sobre el resto de los componentes no plásticos. La Tabla 2 ilustra los resultados porcentuales obtenidos, corroborados posteriormente con las cuantificaciones relativas en microscopio

\footnotetext{
5 De acuerdo a los grados de esfericidad propuestos por Barraclough (1992). 


\begin{tabular}{|c|c|c|c|c|c|c|c|c|c|c|}
\hline Identificación & Sitio & $\begin{array}{c}\text { Matriz } \\
(\%)\end{array}$ & $\mathbf{M v}(\%)$ & $\mathrm{Bt}(\%)$ & $\mathrm{Qz}(\%)$ & $\begin{array}{l}\text { Incl.pum. } \\
\qquad(\%)\end{array}$ & $\begin{array}{c}\mathrm{Pl} / \mathrm{FK} \\
(\%)\end{array}$ & $\mathbf{L t}(\%)$ & $\begin{array}{c}\text { IA / TM } \\
(\%)\end{array}$ & $\begin{array}{c}\text { Rest. org. } \\
(\%)\end{array}$ \\
\hline $\mathrm{LC}_{54 \mathrm{a} 2.1}$ & LCZVIIIS $_{1}$ & 80 & 1.78 & 3.11 & 3.56 & 11.56 & 0.00 & 0.00 & 0.00 & 0.00 \\
\hline $\mathrm{LC}_{54} \mathrm{al}_{62}$ & 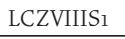 & 80 & 1.78 & 3.11 & 2.67 & 11.11 & 0.00 & 0.89 & 0.00 & 0.44 \\
\hline LC61b29 & 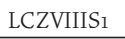 & 80 & 1.27 & 1.59 & 4.44 & 12.06 & 0.00 & 0.32 & 0.32 & 0.00 \\
\hline LC61b8 & 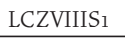 & 80 & 1.93 & 0.65 & 8.39 & 8.39 & 0.00 & 0.65 & 0.00 & 0.00 \\
\hline LC61b55 & 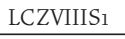 & 70 & 1.84 & 3.67 & 5.51 & 18.37 & 0.00 & 0.61 & 0.00 & 0.00 \\
\hline LC61b59 & LCZVIIIS1 $_{1}$ & 80 & 2.17 & 1.74 & 2.17 & 12.61 & 0.00 & 1.30 & 0.00 & 0.00 \\
\hline $\mathrm{LC}(1)_{117}$ & $\mathrm{LC}(1)$ & 80 & 0.89 & 0.89 & 2.22 & 15.56 & 0.00 & 0.44 & 0.00 & 0.00 \\
\hline $\mathrm{LC}(1)_{94}$ & $\mathrm{LC}(1)$ & 80 & 1.63 & 1.40 & 2.79 & 13.95 & 0.00 & 0.23 & 0.00 & 0.00 \\
\hline $\mathrm{LC}(1) 705$ & $\mathrm{LC}(1)$ & 80 & 3.64 & 5.45 & 1.82 & 9.09 & 0.00 & 0.00 & 0.00 & 0.00 \\
\hline $\mathrm{LC}(1) 250$ & $\mathrm{LC}(1)$ & 90 & 0.62 & 1.60 & 1.23 & 6.54 & 0.00 & 0.00 & 0.00 & 0.00 \\
\hline $\mathrm{LC}(1)_{23}$ & $\mathrm{LC}(1)$ & 90 & 0.85 & 2.34 & 0.85 & 5.96 & 0.00 & 0.00 & 0.00 & 0.00 \\
\hline $\mathrm{LC}(1)_{19}$ & $\mathrm{LC}(1)$ & 90 & 1.13 & 1.45 & 0.48 & 6.45 & 0.00 & 0.32 & 0.16 & 0.00 \\
\hline $\mathrm{LC}(1) 8$ & $\mathrm{LC}(1)$ & 80 & 2.19 & 2.5 & 2.19 & 13.13 & 0.00 & 0.00 & 0.00 & 0.00 \\
\hline $\mathrm{LC}(1)_{740}$ & $\mathrm{LC}(1)$ & 80 & 1.50 & 2.80 & 1.50 & 13.98 & 0.00 & 0.22 & 0.00 & 0.00 \\
\hline
\end{tabular}

Tabla 2. Valores porcentuales de inclusiones no plásticas (Mv: Muscovita; Bt: Biotita; Qz: Cuarzo; Incl. pum.: Inclusiones pumíceas; Pl/FK: Plagioclasa/Feldespato potásico; Lt: Litoclastos; IA/TM: Inclusiones arcillosas/tiesto molido; Rest. org.: Restos orgánicos.

petrográfico. En la mayor parte de los casos los porcentajes van de 10 a $20 \%$, siendo bastante altos si pensamos que la densidad de inclusiones de las pastas no supera el 30\%, correspondiendo la mayor parte de la muestra a densidades de $20 \%$. Microscópicamente confirmamos que las inclusiones de cuarzo, mica y plagioclasa, son los componentes que siguen en abundancia a los fragmentos pumíceos.

Es importante mencionar que el porcentaje total de inclusiones no plásticas en estos fragmentos es bastante superior al que registramos en otros fragmentos del mismo estilo pero sin componentes piroclásticos. La contrastación visual con gráficos mostró que la mayor parte de la muestra $(n=15)$ presenta una relación de $20 \%$ de inclusiones no plásticas y $80 \%$ de matriz. De los casos restantes, tres tienen una densidad de inclusiones de $10 \%, y$ dos de $30 \%$.

\section{$*$ Características generales de las pastas con INCLUSIONES PUMÍCEAS}

Además de describir los componentes pumíceos - que son los primeros indicadores del aporte piroclástico-es necesario considerar los atributos generales de estas pastas, tanto en sus características composicionales como las relaciones presentes entre sus distintas fases (arcilla, inclusiones no plásticas y espacios vacíos). En este sentido, se obtuvo una importante visión de conjunto para abordar la práctica de incorporar las pómez a las pastas. Una de las interrogantes, era si el mineral había llegado individualmente o si había sido incorporado como parte de un depósito que incluía otros componentes.

Con el análisis microscópico identificamos una serie de componentes minerales accesorios no detectados en lupa, representados en proporciones muy inferiores al resto de los componentes, incluso, en muchos casos, en forma aislada en los cortes. Estos fueron turmalina, anfíbol, piroxeno y epidoto. Valores algo mayores corresponden a los litoclastos, pudiendo reconocerse

$\mathrm{N}^{\circ} 38 / 2009$

ESTUdios ATACAMEÑOS

Arqueología y Antropología Surandinas 
un origen variado, metamórfico, plutónico, volcánico y sedimentario, con tamaños y formas variables, aunque mayoritariamente subangulares y subredondeadas. Las plagioclasas (macla albita y karlsbad) y los feldespatos (ortosa y microclino), están presentes en proporciones significativas, al igual que las láminas micáceas, cristales de cuarzo e inclusiones pumíceas.

Así, se vieron dos tendencias en el tamaño de los cristales de las pastas analizadas: una está dada por la fase mineral donde predominan los valores inferiores a $0.25 \mathrm{~mm}$; y la otra está representada por las inclusiones pumíceas con valores entre 0.25 y $1 \mathrm{~mm}$. Esto marca una tendencia general en todos los cortes analizados, remarcando nuevamente la significativa homogeneidad de esta variable. Lo mismo puede decirse respecto de las formas registradas, donde si bien hay clastos angulosos y redondeados, la mayoría presenta un redondeamiento medio que se traduce en formas subangulares y subredondeadas.

La densidad de poros y cavidades, variables en la morfología y orientación a lo largo de la superficie, fue llamativamente alta, aspecto que no habíamos observado en otros análisis de pastas de fragmentos estilísticamente similares (Páez 2005). Como puede observarse en las Figuras 5 a y 5 b, los espacios vacíos suelen estar conectados desde una inclusión a otra. Esta característica, posiblemente vinculada al coeficiente de expansión térmica del sílice, es apropiada en términos de incrementar la resistencia a la ruptura de las piezas, ya que las fracturas al interior de la pasta encuentran en esta "red" de espacios vacíos la vía para disipar, bifurcar y detener la energía (Tite et al. 2001).

Del análisis de los atributos genéticos, morfológicos y tamaños de los componentes cristalinos que acompañan a las pómez en los fragmentos analizados, surgió la posibilidad de que integren el mismo depósito. Los litoclastos representan, tal vez, el componente que menos se adecuaría a esta interpretación, particularmente porque encontramos variados orígenes que excederían el aporte netamente volcánico (líticos metamórficos, plutónicos y sedimentarios). Sin embargo, la escasa proporción presente indicaría que podrían derivar del conducto o del cráter del volcán, o bien, ser incorporados al depósito en la fase de transporte. Esto último también es avalado por las formas redondeadas o subredondeadas registradas en los cortes.

\section{* Naturaleza y origen del aporte piroclástico}

Los datos presentados resaltan la importancia del aporte piroclástico en las pastas. Abundante y homogéneo, parece representar un tipo de recurso ${ }^{6}$ que no pasó inadvertido para las sociedades cuya cerámica es motivo de este análisis. A partir de esto es importante determinar la forma en que estos componentes fueron incorporados en la cerámica, su carácter fortuito o intencional, como una vía para adentrarnos en sus posibles significados sociales. Retomando los resultados del análisis de pasta, preliminarmente parecen posibles tres alternativas sobre su incorporación: a) recolección, molienda e incorporación a la pasta de fragmentos pumíceos y cristales; b) utilización de depósitos epiclásticos que contienen pómez y cristales, y c) utilización de depósitos piroclásticos.

La primera posibilidad requiere recolectar pómez de un depósito piroclástico primario o retrabajado. Cualquiera sea la fuente, previo a la incorporación a las pastas, el material tuvo que ser molido a un tamaño de partículas inferior a $1 \mathrm{~mm}$ para lograr el grado de uniformidad observado en los fragmentos de pómez de las cerámicas, liberando de esta manera el vidrio y la fracción cristalina. Sin embargo, el proceso de molido de materiales para su incorporación en las pastas dará como resultado componentes con una elevada angulosidad debido al modo de rotura del material. Ese es el primer, y tal vez más importante indicador a tener en cuenta. Las observaciones realizadas, tanto a nivel submacroscópico como microscópico, resaltan el carácter redondeado a subredondeado de estas inclusiones, sin aristas visibles, que podamos vincular a la rotura mediante fuerza mecánica humana. Por estas razones esta alternativa interpretativa es poco viable.

La segunda posibilidad considera la utilización de arcillas que contengan material piroclástico. Aunque se han descrito depósitos con ambos componentes ${ }^{7}$, el grado de selección de las pómez no concuerda con lo observado en la cerámica. Esta alternativa se descarta debido a que no es común en el registro geológico la existencia de ar-

\footnotetext{
6 Utilizado este término en su sentido más estricto, como procedimiento o medio, superando sus connotaciones economicistas.

7 Por ejemplo, Arnosio (1995) describe en el Complejo Volcánico de Pocho (prov. de Córdoba), conglomerados formados por fragmentos pumíceos, líticos volcánicos y de basamento igneometamórfico, contenidos en una matriz de granulometría arcillosa.
} 

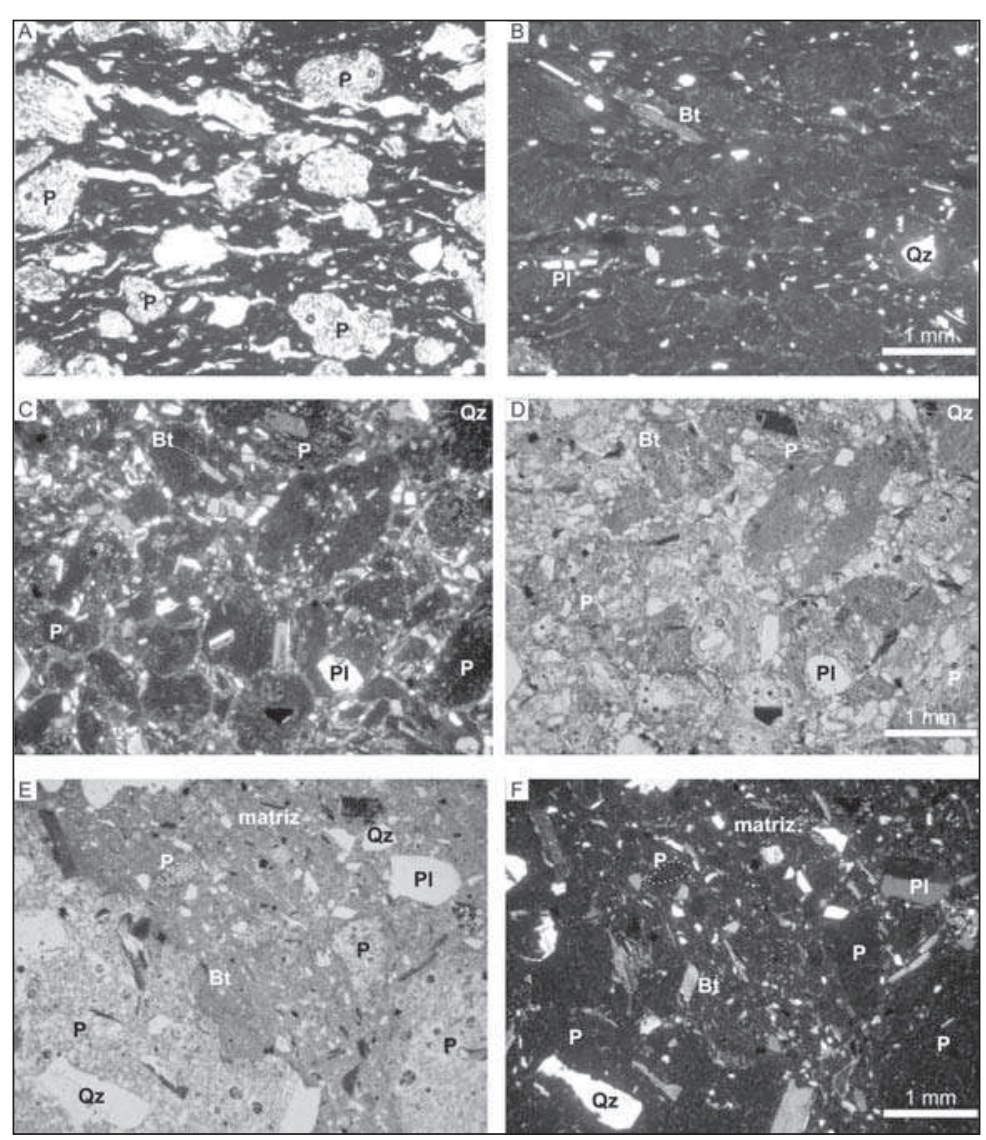

Figura 5. a y b) Corte delgado de cerámica (fragmento LC(1)94) con luz paralela y nicoles cruzados, respectivamente. Se observan fragmentos de pómez ( $\mathrm{P}$ ) y cristales de plagioclasa $(\mathrm{Pl})$, cuarzo $(\mathrm{Qz})$ y biotita $(\mathrm{Bt})$ contenido en la pasta; c y d) Corte delgado de un depósito piroclástico de caída distal, ubicado en las cercanías de Payogastilla (Salta). Está constituido por abundantes fragmentos subredondeados de pómez (P), conformando una fábrica clasto soportada y cristales de plagioclasa (Pl), cuarzo $(\mathrm{Qz})$ y biotita $(\mathrm{Bt}) ; \mathbf{e}$ y $\mathbf{f})$ Corte delgado de ignimbrita formada por fragmentos de pómez $(\mathrm{P})$ de tamaño y formas variables, cristales de plagioclasa $(\mathrm{Pl})$, cuarzo $(\mathrm{Qz})$ y biotita $(\mathrm{Bt})$ inmersos en una matriz de grano muy fino, compuesta por vidrio volcánico; $\mathbf{a}, \mathbf{c}$ y $\mathbf{e})$ Nicoles paralelos; $\mathbf{b}, \mathbf{d}$ y $\mathbf{f}$ ) Nicoles cruzados.

cillas aptas para la manufactura cerámica que contengan: 1) fragmentos de pómez con una notable uniformidad en el tamaño ( 0.25 a $1 \mathrm{~mm}) ; 2$ ) un elevado grado de preservación del vidrio volcánico, el cual no está alterado, y 3 ) fragmentos de cristales de plagioclasa, cuarzo y biotita, como para constituir la materia prima para la elaboración de cerámica, sin mediar procesamiento previo (molienda, tamizado, selección manual, entre otros).

La tercera alternativa es aquella en la cual las pómez y cristales provienen de un depósito piroclástico. Existen tres grandes grupos de depósitos piroclásticos que contienen pómez y fragmentos de cristales como los observados en la cerámica: a) depósitos de flujos piroclásticos; b) depósitos de oleadas piroclásticas, y c) depósitos piroclásticos de caída, también conocidos como "lluvia de tefra". Dentro de aquellos de flujos piroclásticos, las ignimbritas son los principales depósitos que contienen pómez y cristales. Están formados por ceniza (generalmente $>50 \%$ ), fragmentos pumíceos y proporciones variables de cristales y líticos. Se caracterizan por el escaso a nulo grado de selección por tamaño y densidad de sus

$\mathrm{N}^{\circ} 38 / 2009$

EsTUdios ATACAMEÑOS

Arqueología y Antropología Surandinas 
componentes (Cas y Wright 1987), originándose a partir de erupciones volcánicas explosivas que forman columnas eruptivas que pueden colapsar y generar flujos piroclásticos con una elevada concentración de partículas. La distancia alcanzada por los flujos piroclásticos es variable ( $<1 \mathrm{~km}$ a $200 \mathrm{~km}$ ) y los volúmenes oscilan entre $<0.1 \mathrm{~km}^{3}$ hasta $5000 \mathrm{~km}^{3}$, dependiendo principalmente de la intensidad de la erupción y del tipo de aparato volcánico que los generó.

Las ignimbritas tienen una amplia distribución y volumen en la cordillera de los Andes, con una asociación mineral formada por plagioclasa, cuarzo y biotita como las fases más abundantes. Los depósitos de esta naturaleza más cercanos a los sitios arqueológicos estudiados, corresponden a la Ignimbrita Pucarilla (Hongn y Seggiaro 2001 ), situada a $85 \mathrm{~km}$, y a la ignimbrita asociada a la caldera del cerro Galán (Sparks et al. 1985), ubicada a 120 km. González (1990) definió el Complejo Volcánico Portezuelo Las Ánimas, emplazado en el faldeo occidental de la sierra de Aconquija, aproximadamente a $35 \mathrm{~km}$ de los sitios estudiados. Este autor describió una sucesión de $540 \mathrm{~m}$ de potencia formados por brechas piroclásticas, brechas lapillíticas y escasos niveles de tobas. Sin embargo, no menciona fragmentos pumíceos en ninguno de los depósitos que constituyen este complejo volcánico.

Los depósitos de oleadas piroclásticas se originan en erupciones generadas por la interacción del magma con agua (acuífero, río, laguna, etc.). La distancia alcanzada no supera los $10 \mathrm{~km}$ desde el centro de emisión. En comparación con las ignimbritas, estos depósitos son mejor seleccionados, pudiendo contener proporciones variables de fragmentos pumíceos, cristales y líticos. Se asocian a aparatos volcánicos denominados maar y a anillos de tobas. En la cordillera de los Andes, este tipo de aparatos volcánicos y sus depósitos asociados no son frecuentes y suelen estar subordinados a las ignimbritas. Por otra parte, los depósitos de caída son originados por erupciones volcánicas explosivas. El material piroclástico es eyectado por el conducto del volcán y asciende en la atmósfera formando una columna eruptiva, que puede alcanzar hasta $50 \mathrm{~km}$ de altura, dependiendo de la intensidad de la erupción. El material piroclástico que forma la mayor parte de la columna eruptiva es de granulometría fina, tamaño ceniza ( $<2 \mathrm{~mm}$ ) o lapilli fino, el cual es dispersado a decenas, cientos o miles de kilómetros desde el volcán por la influencia de los vientos atmosféricos regionales. Esto causa un efecto de selección natural por tamaño y densidad de los componentes emitidos, que es más efectivo mientras mayor es la distancia desde el centro de emisión. El material dispersado finalmente comienza a precipitar por la gravedad, depositándose uniformemente tanto en las partes altas como en los sectores más bajos del terreno, inclusive en lagos y lagunas. Estos depósitos se componen principalmente de ceniza volcánica, fragmentos de cristales correspondientes al magma que formó la erupción, fragmentos de pómez y proporciones variables de fragmentos líticos de diversa composición. En términos generales, los depósitos de caída son inconsolidados, aunque pueden estar ligera o intensamente litificados. Poseen una amplia distribución en el Noroeste Argentino, desde la Puna hasta las sierras subandinas y representan la acumulación en facies distales del volcanismo calcoalcalino explosivo desarrollado principalmente durante el Terciario MedioCuaternario (Pereyra et al. 2008). Los estudios mineralógicos realizados señalan el predominio de plagioclasa, cuarzo y biotita, además de trizas vítreas y fragmentos pumíceos (Pereyra com. pers. 2008).

\section{* Ignimbritas o depósitos De CAída}

Como ya mencionamos, el rasgo característico de las inclusiones es la uniformidad en el tamaño y abundancia o proporción modal de los fragmentos pumíceos y de los cristales, así como la escasa presencia de fragmentos líticos que no son de origen volcánico. A partir de esto, y en comparación con los resultados de los análisis submacroscópicos y microscópicos realizados en las pastas, la tercera alternativa propuesta -incorporación de un depósito piroclástico- es la que más se ajusta a nuestras observaciones. Sin embargo, como dijimos, dentro de esta categoría hay tres tipos de depósitos posibles.

Los depósitos de oleada tienen una distribución y abundancia muy acotada en el Noroeste Argentino, por lo que parecen ser los menos probables de los tres. De esta manera, nuestras opciones se redujeron a los depósitos de flujo (ignimbritas) y a los depósitos de caída. A estos fines fue útil la comparación de las características petrográficas de ignimbritas y tobas con lo observado en las pastas cerámicas. 
En las Figuras 5 e y $5 \mathrm{f}$ se muestran las características petrográficas de una ignimbrita formada por fragmentos pumíceos, cristaloclastos de plagioclasa, cuarzo y biotita y un agregado de grano muy fino, compuesto por vidrio volcánico. Destaca el elevado porcentaje de ceniza de grano muy fino y la variación en el tamaño y forma de los fragmentos pumíceos, rasgos característicos de las ignimbritas. Los rasgos texturales de un depósito de caída primario (es decir, que no ha sufrido procesos de removilización luego de la depositación, por viento o agua, que modifiquen la textura primaria $y / 0$ que incorporen componentes ajenos al material original depositado) pueden observarse en las Figuras 5 c y $5 \mathrm{~d}$. Hay un alto contenido de fragmentos pumíceos, con una elevada selección por tamaño y prácticamente en contacto mutuo entre ellos. También destacan los cristales fragmentados de plagioclasa, cuarzo y biotita, de menor tamaño que las pómez, ocupando los espacios entre éstas y el escaso material vítreo de grano muy fino.

A partir de la comparación de los rasgos texturales de la ignimbrita con las cerámicas, no se observa una concordancia en el tamaño de las pómez (Figura 5), así como componentes vítreos de tamaño ceniza incorporados a la pasta. Estas características implican asumir una serie de consideraciones para su incorporación: 1) que los fragmentos pumíceos fueron previamente seleccionados por tamaño, en el rango observado en la cerámica ( $<1$ $\mathrm{mm})$; 2) que el depósito carecía de fragmentos líticos o su proporción era muy baja, 7\% (ver Tabla 2) o que este componente haya sido removido manualmente, previo a la incorporación, y 3) que el material vítreo de grano muy fino, está cubierto por la pasta de color rojizo, impidiendo su reconocimiento.

Cuando se comparan los rasgos texturales de las inclusiones y cristales presentes en la cerámica, con aquellos del depósito de caída, se observa una notable similitud en la forma, tamaño y contenido de cristales de los fragmentos pumíceos, así como en las fases minerales presentes (plagioclasa, cuarzo y biotita) y la morfología fragmentada que poseen.

Entonces, los depósitos de caída se ajustan en mayor grado al tipo de aporte piroclástico presente en las pastas cerámicas analizadas que habría funcionado como antiplástico. Esto de acuerdo a: 1) El modo natural de selección por tamaño y densidad, constituye una fuente de material antiplástico para la cerámica estudiada, ya que no es necesario ningún procesamiento previo, como molido, selección manual, etc.;2) Estos depósitos se presentan generalmente inconsolidados o con una ligera litificación, lo que facilita su extracción; 3) La composición mineralógica similar a la observada en las cerámicas, y 4) La amplia distribución de los depósitos de caída en el Noroeste Argentino, sugiere una alta probabilidad de contar con uno cercano, por lo cual no habrían sido necesarios grandes desplazamientos, como en el caso de considerar una ignimbrita como fuente.

\section{* Prácticas de manufactura}

A través del análisis de la naturaleza y características de las inclusiones blancas presentes en las pastas cerámicas, procuramos aproximarnos a la forma en que estas piezas fueron manufacturadas y a las prácticas involucradas en esta parte del proceso productivo. Como mencionamos, los depósitos de caída son originalmente inconsolidados, es decir, se pueden separar manualmente, aunque no podemos asumir que este sea efectivamente el estado de los depósitos al momento de su extracción para la manufactura cerámica, dado que el paso del tiempo es un importante agente que interviene en el estado original de los depósitos, pudiendo otorgarle cierto grado de cohesión a sus componentes. Sin embargo, se pueden encontrar bastante deleznables.

Las pómez de las pastas cerámicas presentan un grado de angulosidad muy bajo, similar al observado en los depósitos de caída, por lo que podríamos pensar entonces en la posibilidad de que si bien el depósito pudiera tener cierto grado de consolidación, el material era fácilmente extraíble, quizás en trozos, y la disgregación final no requería una fuerza mecánica importante. Este último proceso técnico podría tener lugar en dos momentos diferentes de la manufactura. Una posibilidad es que los depósitos sean disgregados manualmente a través de la aplicación de una fuerza mecánica muy suave que no implique la rotura de los componentes individuales, generando formas angulosas. Esto se realizaría antes de la incorporación en el cuerpo arcilloso, y por ende, a la mezcla de los componentes y al amasado. 
La otra alternativa es que el proceso de disgregación se produzca durante el amasado. El depósito de caída sería incorporado en trozos a la matriz plástica, separándose los componentes durante el proceso de mezcla y amasado. Para facilitar la división, las fracciones incorporadas no podrían ser de gran tamaño. En este sentido, el depósito de caída con el que comparamos la cerámica se disgregaba al solo contacto con el agua, sin que necesitara mediar ningún proceso adicional de fragmentación.

De cualquier manera, las dos alternativas requieren un proceso previo de selección y extracción del depósito, además de su eventual transporte al lugar de manufactura. Con la información que tenemos es difícil hipotetizar en torno a la manufactura local o externa de la cerámica con inclusiones pumíceas, para lo cual necesitaríamos contar con análisis específicos de procedencia. Sin embargo, un dato a considerar es la disponibilidad local de este tipo de recursos. En la región se encuentran rocas piroclásticas, principalmente tobas de edad terciaria, sobre las que se asientan sedimentitas loésicas cuaternarias con intercalaciones de bancos de tobas y chonitas que se distinguen en todo el valle, conformando un paleoambiente lacustre de depositación de lluvias de cenizas (Fernández 1995 Ms). Tenemos presente que la disponibilidad local de los recursos empleados en la manufactura cerámica (arcilla y material piroclástico) no asegura que las piezas cuyos fragmentos analizamos sean efectivamente manufacturadas en el valle. En este sentido, la movilidad tanto de bienes como de personas era una de las características de la política incaica que habría contribuido a configurar las relaciones entre el Estado y las poblaciones locales (Rostworowski 1999 [1988]).

La proporción de material piroclástico incorporado, que reuniría tanto a las pómez como a la fracción clástica más fina, habría alcanzado proporciones variables, entre 10 y $30 \%$, aunque en la mayor parte de los casos predominan valores de $20 \%$, sugiriendo ciertas normas en la adición. En otro conjunto cerámico, también procedente del valle, pudimos confirmar estos porcentajes (Manasse y Páez 2008), llevándonos a considerar la posibilidad de cierta estandarización en el proceso de preparación de estas pastas para la cerámica de Tafí.

\section{* Consideraciones finales}

Hemos analizado un aspecto puntual vinculado a la forma en que se elaboran estas piezas, la naturaleza y características de las inclusiones piroclásticas, y el proceso técnico por el cual habrían sido incorporadas en las pastas. Resultó clara la importancia del aporte piroclástico, particularmente a partir de la incorporación de depósitos de caída, a los cuales corresponderían no solo las inclusiones pumíceas reconocidas en las pastas, sino también la fracción mineral más fina como cuarzos, micas, feldespatos, plagioclasas y otros, así como los escasos litoclastos registrados.

La dispersión atmosférica de este material favorece su disponibilidad en regiones que no manifiestan ni manifestaron actividad volcánica, como es el caso del valle de Tafí. La presencia de niveles de tobas, tal como se desprende de estudios geológicos, permite plantear la posibilidad de que se hayan utilizado recursos locales para la manufactura cerámica. En este sentido, será útil correlacionar el aporte piroclástico presente en las pastas y la mineralogía de los depósitos de caída locales a partir de datos composicionales.

También es importante tener presente el aporte piroclástico a nivel regional, en vista de la magnitud de esta manifestación en el Noroeste Argentino. Esta práctica, vinculada a la presencia incaica, habría tenido connotaciones importantes para la política del Tawantinsuyo en esta región. Un dato a considerar es la presencia de estos materiales no solamente en formas incas diagnósticas, sino también en alfarería de momentos previos, como aquella de los estilos Santa María y Belén. Esto podría mostrar la participación de la población local del valle en las prácticas sociopolíticas vinculadas al uso de esta materia prima.

Es posible que su uso responda, en parte, a un interés por disminuir el peso de las piezas y hacerlas más resistentes. La baja densidad de las pómez, combinado con la resistencia a la fractura dada por la abundancia de cavidades generadas a partir de la expansión-contracción del sílice sujeto a variaciones térmicas, da como resultado objetos factibles de transportar. Es importante evaluar, sin embargo, en qué marco de demandas 
sociales o sociopolíticas se da la necesidad de contar con piezas que puedan ser movilizadas con más facilidad y menos posibilidad de que se rompan. En este sentido, se deben tener en cuenta las políticas negociativas que involucran la circulación de piezas, como es el caso de prácticas de intercambio o regalos, o bien, aquellas orientadas al reforzamiento de los lazos entre las poblaciones locales y el Estado que se vinculan a contextos festivos.

Más allá de las implicancias funcionales, no podemos obviar la necesidad de buscar el significado de estas inclusiones a partir del interés en reforzar la idea de pertenencia a un nuevo orden social y político, situación que podría materializarse a través de la implementación regulada de un conjunto de pautas culturales, en este caso vinculadas a la forma de manufacturar los bienes alfareros.

Sea cual sea el caso, es clara la intención de redefinir las características de la producción alfarera durante momentos incaicos, extendiéndose tanto a las formas estatales más diagnósticas como a las que se asocian al Tardío local. La presencia de estas últimas nos lleva a la posibili- dad de que la producción de estilos locales e incas no se produzca de manera separada e independiente.

Otro aspecto a tener en cuenta es la forma en que las restricciones en el acceso a los recursos piroclásticos podrían condicionar la producción. Estos depósitos no tienen la frecuencia que puede encontrarse en otros componentes de las pastas, como por ejemplo, las arenas, cuya disponibilidad local está sujeta a la presencia de cursos de agua. Es necesario considerar, entonces, la posibilidad de que la restricción en la disponibilidad genere puntos de manufactura específicos a partir de los cuales las piezas con inclusiones alcanzarían las distintas localizaciones estatales; o bien, que se favorezca la circulación de las materias primas piroclásticas entre los distintos lugares de manufactura, sean estos locales o regionales.

Las ideas aquí vertidas, lejos de ser concluyentes, para lo cual necesitaríamos un soporte empírico mucho más amplio, pretenden promover la discusión. Las líneas de investigación que estamos desarrollando permitirán profundizar en esta problemática y sus implicancias para la organización de la producción durante el momento de expansión incaica en el Noroeste Argentino.

\section{$*$ Referencias citadas}

AMBROSETTI, J. B., 1908. Exploraciones arqueológicas en la ciudad prehistórica de La Paya. Publicaciones de la Sección Antropología de la Facultad de Filosofia y Letras de la Universidad de Buenos Aires 3.

BARRACLOUGH, A., 1992. Quaternary sediment analysis: A deductive approach at A-level. Teaching Geography 17: 15-18.

BENNETT, W., E. BLEILER y F. SOMMER, 1948. Northwest Argentine archaeology. Publications in Anthropology 38. Yale University Press, Connecticut.

BREGANTE, O., 1926. Ensayo de clasificación de la cerámica del Noroeste Argentino. A. Estrada y Cía. Eds., Buenos Aires.

CAS, R. A. y J. V. WRIGHT, 1987. Volcanic successions. Modern and ancient; a geological approach to processes, products and successions. Allen \& Unwin, Londres.

CREMONTE, B., 1991. Caracterizaciones composicionales de pastas

cerámicas de los sitios Potrero Chaquiago e Ingenio del Arenal Médanos. Shincal 3 (1):33-47.

1994. Las pastas cerámicas de Potrero Chaquiago (Catamarca), producción y movilidad social.Arqueología 4: 133-164.

CREMONTE, M. B., I. L. BOTTO, A. M. DÍAS, R. VIÑA y M. E. CANAFOGLIA, 2007. Vasijas Yavi-Chicha: Distribución y variabilidad a través del estudio de sus pastas. Pacarina número especial, vol. 2, pp. 189-194.

DE LA FUENTE, G., 2007. Producción y tecnología cerámica: Estandarización, especialización y procedencia en Batungasta (valle de Abaucán, depto. Tinogasta, Catamarca, Argentina). Tesis doctoral. Universidad Nacional de La Plata, La Plata.

FERNÁNDEZ, R. I., 1995 Ms. Informe geoindustrial de los depósitos cineríticos del sector oriental del valle de Tafí, provincia de Tucumán, Universidad del Norte Santo Tomás de Aquino, Tucumán. 
GONZÁLEZ, A. R., 1955. Contextos culturales y cronología relativa en el área central del Noroeste Argentino (nota preliminar).Anales de Arqueología y Etnología XI.

1963. Problemas arqueológicos de la Puna argentina. En $A$ Pedro Bosch Gimpera en el septuagésimo aniversario de su nacimiento, pp. 58-72. Instituto Nacional de Antropología e Historia, Universidad Nacional Autónoma de México, México D.F.

GONZÁLEZ, O. E., 1990. Las volcanitas del Portezuelo Las Ánimas, sierra de Aconquija, provincias de Catamarca y Tucumán. Revista de la Asociación Geológica Argentina XLV (3-4):386-396.

HONGN, F. D. y R. SEGGIARO, 2001. Hoja Geológica 2566-III. Cachi. Boletín 248. Programa Nacional de Cartas Geológicas. 1:250.000. SEGEMAR.

KRAPOVICKAS, P., 1975. Algunos tipos cerámicos de Yavi Chico.Ac-

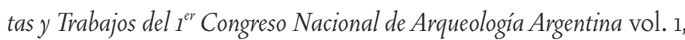
pp. 293-300. Buenos Aires.

MANASSE, B., 1995-96. Proyecto de arqueología de rescate en Los Cuartos, este de Tafí del Valle. Shincal 5: 81-93.

2001. Las evidencias arqueológicas del valle de Tafí: Una mirada desde la gestión de los recursos culturales. XIV Congreso Nacional de Arqueología Argentina. Rosario. En prensa.

2003. Material cerámico del este de Tafí del Valle, provincia de Tucumán: El último milenio de ocupación aborigen en la región. Congreso Regional de Ciencia y Tecnología, NOA. Catamarca. En prensa.

MANASSE, B. y M. C. PÁEZ, 2008. El valle de Tafí en tiempos del Imperio incaico: Inferencias a partir de la tecnología alfarer.Mundo de Antes 5. En prensa.

MANASSE, B., R. OVEJERO y M. C. PÁEZ, 2006. Estudios tecnológicos de alfarería tardía del este del valle de Tafí, Tucumán. Actas del XV Congreso Nacional de Arqueología Argentina (2004). Río Cuarto. En prensa.

MARCHEGIANI, M., V. PALAMARCZUK y A. REYNOSO, 2007. El estilo como frontera. Sobre las urnas Negro sobre Rojo de momentos tardíos de Yocavil (Noroeste Argentino). Resumen Ampliado. XVI Congreso Nacional de Arqueología Argentina vol. 2, pp. 451-456. San Salvador de Jujuy.

PÁEZ, M. C., 2005. El Tardío en el valle de Tafí, provincia de Tucumán: Estudio de material alfarero santamariano. Tesis de Licenciatura. Escuela de Arqueología, Universidad Nacional de Catamarca, Catamarca.
2008 Ms. De presencias y ausencias. Cambios y continuidades en la tecnología alfarera de las sociedades del valle de Tafí.

PÁEZ, M. C. y C. J. PATANÉ, 2007. Análisis tecnológico de alfarería incaica: Los platos pato del Pucara de Las Lomas Verdes (Tafí del Valle, Tucumán). Aportes Cientificos desde Humanidades, pp. 283296. Facultad de Humanidades, Universidad Nacional de Catamarca, Catamarca.

PÁEZ, M. C. y M. GIOVANNETTI, 2008. Intersecciones y síntesis. Sincretismos en los platos del período incaico en el Noroeste Argentino. Arqueología Suramericana 4 (2): 169-190.

PATANÉ, C. J., 2008. Arqueología de los encuentros: Lo inca y lo local en un sitio en las montañas de un valle del NOA. Estudiando al Pucara de las Lomas Verdes (Tafí del Valle, prov. de Tucumán). Tesis de Licenciatura. Universidad Nacional de Catamarca, Catamarca.

PEREYRA, R., R. BECCHIO, R. HERNÁNDEZ, J. G. VIRAMONTE y A. SCHULTZ, 2008. Utilización de niveles piroclásticos como niveles de guía cronoestratigráficos en la industria del petróleo, sierras subandinas, noroeste de Argentina. IX Congreso Geológico de América Central, pp. 124. San José de Costa Rica.

PERROTA, E. y C. PODESTÁ, 1973 Ms. Aplicación de la técnica de seriación a la colección de urnas y pucos santamarianos provenientes del valle de Yocavil. Ponencia presentada al III Congreso Nacional de Arqueología Argentina, Salta.

1978. Contribution to the San José and Santa María cultures, Northwest Argentina. En Advances in Andean archaeology, D. Browman (Ed.), pp. 525-551. Mouton, The Hague, París, Chicago.

PUENTE, V., 2008 Ms. Caracterización tecnológica y composicional de alfarería Belén. Un abordaje desde la petrografía cerámica. Ponencia presentada a las Jornadas sobre Avances en Técnicas Analíticas Aplicadas al Estudio de Materiales en Arte y Arqueología, Buenos Aires.

RAFFINO, R., 2004. El Shincal de Quimivil. Editorial Sarquís, Catamarca.

ROSTWOROWSKI, M., 1999 [1988]. Historia del Tahuantinsuyo. Instituto de Estudios Peruanos, Lima.

SANTILLÁN DE ANDRÉS, S., 1951. Poblaciones indígenas en el valle de Tafí. Geographia una et varia. Universidad Nacional de Tucumán, Tucumán.

SCHIAPPACASSE, V., V. CASTRO y H. NIEMEYER, 1989. Los desarrollos regionales en el Norte Grande (1000-1400 DC). En Culturas de Chile. Prehistoria. Desde sus orígenes a los albores de la Conquista, J. Hidalgo, V. Schiappacasse, H. Niemeyer, C. Aldunate e I. Solimano (Eds.), pp. 181-220. Editorial Andrés Bello, Santiago. 
SEMPÉ, C., 1999. La cultura Belén. Actas del XII Congreso Nacional de Arqueología Argentina vol. 2, pp. 250-258. La Plata.

2006. El período Tardío en Azampay: El señorío Belén y su modelo geopolítico. En Azampay. Presente y pasado de un pueblito catamarqueño, C. Sempé, S. Salceda y M. Mafia (Eds.), pp. 365-380. Ediciones al Margen, Buenos Aires.

SOLÁ, P. y M. MORALES, 2007. Caracterización petrográfica y biológica de un tiesto cerámico estilo "incaico" hallado en Susques (Jujuy). Intersecciones en Antropología 8:361-364.

SPARKS, R., P. FRANCIS, R. HAMER, R. PANKHURST, L. O'CALLAGHAN, R. THORPE y D. R. PAGE, 1985. Ignimbrites of the cerro Galan caldera, NW Argentina. Journal of Volcanolgy and Geothermal Research 24: 205-248.
TARRAGÓ, M., 2000. Chacras y pucara. Desarrollos sociales tardíos. Nueva Historia argentina. vol. 1. Los pueblos originarios y la Conquista, M. Tarragó (Ed.), pp. 257-300. Editorial Sudamericana, Buenos Aires.

TITE, M., V. KILIKOGLOU y G. VEKINIS, 2001. Review article. Strength, touchness and thermal shock resistance of ancient ceramics, and their influence on technological choice. Archaeometry $43(3): 301-324$

WEBER, R., 1978. A seriation of the late prehistoric Santa María culture of Northwestern. Fieldiana Anthropology 68 (2):49-98.

WILLIAMS, V., 2003 Ms. Poder y cultura material bajo el dominio inca. Ponencia presentada al $1^{\text {er }}$ Congreso de Historia Sudamericana, Santa Cruz de la Sierra. 